\title{
CARACTERÍSTICAS DE LATOSSOLOS ROXOS DESENVOLVIDOS DE ROCHAS ALCALINAS E BÁSICAS DE JABOTICABAL, SP'
}

\author{
J.F. CENTURION²; I. ANDRIOLI'; J. MARQUES Jr. ${ }^{2}$; D. G. MARCHIORI \\ ${ }^{2}$ Departamento de Solos e Adubos - FCAV/UNESP, CEP:14870-000, Jaboticabal ,SP. \\ ${ }^{3}$ Pós-Graduando, FESC/USP, São Carlos,SP
}

\begin{abstract}
RESUMO: Comparou-se as características químicas, fisicas e mineralógicas de Latossolos Roxos desenvolvidos de rocha alcalina (LRe) e de rocha eruptiva básica (LRd), da regiāo de Jaboticabal, SP. Os resultados indicaram que o LRe apresenta-se num estádio de desenvolvimento, comparativamente, menos evoluido que o LRd, enquanto esse se apresenta com tendência para a maturidade. Os menores teores de argila e ferro total e maiores teores de saturação por bases, Kl e CTC da fraçāo argila apresentados pelo LRe comprovam esta tendência. Conclứ-se que a ocorrência de Latossolo Roxo eutrófco no campus da UNESP-Jaboticabal, pode estar associada a participação de rochas alcalinas na sua pedogênese.
\end{abstract}

Descritores: latossolo roxo, rocha alcalina, pedogênese

\section{CHARACTERISTICS OF DUSKY RED LATOSOLS DEVELOPED FROM ALCALINE AND BASIC ROCKS IN JABOTICABAL, SP}

SUMMARY: A comparative study was carried out on chemical, physlcal and mineralogical characteristics of dusky red latosols originated from the weathering of alcaline (LRe) and basic eruptive rocks (LRd), of the Jaboticabal county, Brazil. The results showed that the LRe presented a younger development stage than LRd, while this soil showed a tendency to maturity. This trend was corraborated through the lower clay and total $\mathrm{Fe}_{2} \mathrm{O}_{3}$ contents and higher base saturation contents, $\mathrm{SiO}_{2} / \mathrm{Al}_{2} \mathrm{O}_{3}$ molecular ratio $(\mathrm{KI})$ and clay fration $\mathrm{CEC}$ presented by $\mathrm{LRe}$. It can be conchuded that the occurrence of eutrophic dusky red latosols in the UNESP-Jaboticabal campus may be due to the influence of alcaline rocks.

Key Words: dusky red latosol, alcaline rocks, pedogenesis

\section{INTRODUÇÃO}

A região de Jaboticabal se destaca na atividade agrícola, como grande produtora de cana-deaçúcar $\mathrm{e}$ amendoim. $\mathrm{A}$ adoção de tecnologias de ponta nas práticas culturais relativas ao manejo do solo cultivados com estas culturas é facilitada pela ocorrência na região de solos situados em relevo ${ }^{\prime}$ plano ou suave ondulado. Dentre eles o de maior ocorrência segundo o CENTRO NACIONAL DE ENSINO E PESQUISAS AGRONÔMICAS COM SOLOS (1960) é o Latossolo Roxo. Os autores relatam como material de origem desse solo as rochas provenientes do diabásio, ou seja, as eruptivas básicas constituem o material que deu origem aos solos desta unidade de mapeamento.

OLIVEIRA \& MENK (1984) descrevendo sobre os Latossolos Roxos do Estado de São Paulo citam que basalto e diabásio constituem as principais rochas que originam o Latossolo Roxo. Os au- tores consideram que o basalto, pela extensão que ocupam, a rocha mais importante na gênese desse solo.

Embora as rochas eruptivas básicas apresentem composição variada, a presença de piroxênios e feldspatos é comum (GUERRA, 1969 e ROTTA, 1972). Este último autor define rochas alcalinas como aquelas constituídas de minerais em cuja composição química a relação $\mathrm{SiO}_{2} /\left(\mathrm{K}_{2} \mathrm{O}, \mathrm{Na}_{2} \mathrm{O}\right)$ é menor que 6. $O$ mesmo relata a ocorrência de maciços alcalinos no Estado de São Paulo, nas regiões da Ilha de São Sebastiăo, Ipanema, Jacupiranga, Cananéia, Passa-Quatro e Itapirapuã.

A ocorrência de rochas alcalinas na região de Jaboticabal foi descoberta e relatada por PINOTTI et al. (1970). Esta rocha foi estudada petrograficamente por GOMES \& VALARELLI (1970) que a consideraram como um tinguaito do terciário. No mapeamento da região de Ribeirão Preto-SP, foi registrado a ocorrência de tinguaitos em

\footnotetext{
'Trabalho realizado com apoio financeiro da Fundação para o Desenvolvimento da UNESP-FUNDUNESP.
} 
Jaboticabal numa área aproximada de $40 \mathrm{Km}^{2}$ (SÃO PAULO, 1974).

COUTINHO et al. (1982) relataram a ocorrência de lavas alcalinas analcimíticas associadas ao grupo Bauru no Estado de São Paulo. Os autores após estudos com microscopia em amostras provenientes da analcima tinguaito de Jaboticabal, consideraram que as mesmas apresentam em ordem de abundância nefelina, sanidina, Na-augita e analcima como essenciais. Ti-augita, magnetita, esfeno e enigmatita são acessórios relativamente abundantes, ocorrendo em menores proporções de acessórios zirconiferos, anfibólio sódico, apatita e perovskita.

Posteriormente, BARCHA et al. (1984) realizaram levantamento magnetométrico no Campus da Faculdade de Ciências Agrárias e Veterinárias, UNESP-Jaboticabal com o objetivo de conhecer melhor a ocorrência de rochas alcalinas ali descritas. Os resultados desse levantamento, aliado aos resultados obtidos por análise petrográficas, permitiram aos autores supor uma manifestação magmática alcalina de amplitude bem maior que aquela até então suposta.

Apesar da existência na literatura de trabalhos que relatam a existência da intrusão alcalina na região de Jaboticabal-SP, os mesmos não estudaram sua influência na formação e propriedades dos solos.

Visando contribuir com informações a respeito de solos oriundos de rochas alcalinas é que se propôs o presente trabalho. Assim, objetiva-se verificar através de descriçðes morfológicas de perfis e análises fisicas, químicas e mineralógicas a influência da rocha alcalina (tinguaito) na gênese do Latossolo Roxo e compará-lo com o Latossolo Roxo, desenvolvido sob a influência de rocha eruptiva básica (basalto).

\section{MATERIAL E METODOS}

Características de meio fisico: O município de Jaboticabal acha-se localizado na parte norte do Estado de São Paulo, apresentando como coordenadas geográficas: $21^{\circ} 15^{\prime}$ de latitude $S, 4^{\circ} 18^{\prime}$ de longitude $W$ e $575 \mathrm{~m}$ de altitude. O clima é do tipo Cwa, isto é mesotérmico de inverno seco. A vegetação primária é do tipo floresta latifoliada tropical. Atualmente a área em estudo encontra-se ocupada pelos campos experimentais da UNESP. $O$ relevo do local é suave ondulado, sendo que do ponto de vista geológico, destaca-se a presença de basaltos da formação Serra Geral (INSTITUTO DE PESQUISAS
TECNOLÓGICAS, 1981) e de rochas alcalinas (GOMES, 1970; PINOTTI et al., 1970; COUTINHO et al., 1982; SÃO PAULO, 1974 e BARCHA el al., 1984).

Amostragem de solo e métodos analíticos: As amostras de solo foram coletadas em trincheiras localizadas no final de uma encosta. As mesmas foram abertas em locais previamente escolhidos, numa tentativa de variar apenas o material de origem (basalto e tinguaito), como fator responsável pela formação do solo. A Figura 1, ilustra a posição das trincheiras, estando estas distanciadas cerca de $1 \mathrm{Km}$. A descrição morfológica do perfil foi feita de acordo com LEMOS \& SANTOS (1982). Os métodos analíticos referentes às análises fisicas e quimicas seguiram as metodologias adotadas por CAMARGO et al. (1986), sendo que a caracterização do teor de fósforo nos perfis foi realizada pelo método da extração com resina trocadora de íons, como descrito por RAIJ \& QUAGGIO (1983).

As análises referentes ao ataque por $\mathrm{H}_{2} \mathrm{SO}_{4}$ $\left(\mathrm{SiO}_{2}, \mathrm{Al}_{2} \mathrm{O}_{3}\right.$ e $\mathrm{Fe}_{2} \mathrm{O}_{3}$ ), foram feitas após digestão com $\mathrm{H}_{2} \mathrm{SO}_{4} 1: 1$ (EMPRESA BRASILEIRA DE PESQUISA AGROPECUÁRIA, 1979). Os óxidos de ferro livres (Fed) foram extraídos com ditionito-citratobicarbonato, segundo MEHRA \& JACKSON (1960). $O$ ferro amorfo foi extraído com oxalato de amônio de acordo com CAMARGO et al., 1986.

A análise térmica diferencial foi usada para a determinação quantitativa da caulinita e gibbsita, presentes na fração argila desferrificada e saturada com magnésio e aquecidas da temperatura ambiente até $700^{\circ} \mathrm{C}$. Foram coletadas amostras no afloramento do tinguaito e realizadas análise petrográfica em lâminas delgadas, observadas no microscópio sob luz polarizada.

\section{RESULTADOS E DISCUSSÃO}

Foram abertas três trincheiras em locais em que o principal material de origem que resultou na formação do solo foi o basalto. Também na mesma encosta (Figura 1), foram descritas, coletadas e analisadas amostras de três trincheiras próximas a um afloramento de tinguaito.

Por se tratar de uma rocha de pouca ocorrência no Estado de São Paulo, e com poucas informações na literatura Brasileira a respeito de seus minerais, foi realizada análise petrográfica de material proveniente deste afloramento alcalino. Os resultados indicaram em ordem de abundância: nefelina, sanidina, analcima, piroxênios, titanita, opacos e 
P1 = Localização das amostragens do Latossolo Roxo Eutrófico (LRe)

P2 = Localização das amostragens do Latossolo Roxo Distrófico (LRd)

P3 = Localização da afloramento da rocha alcalina (tinguaito)

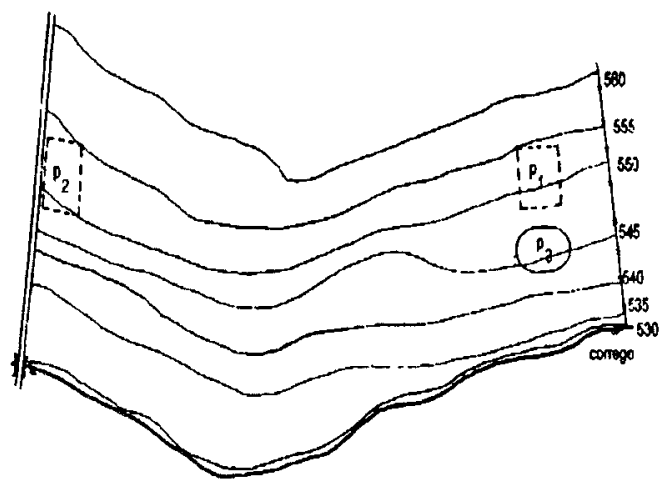

Figura 1 - Detalhe planialtimétrico da vertente onde foram realizadas amostragem.

acessórios, confirmando os estudos feitos em rochas alcalinas da região de Jaboticabal por GOMES \& VALARELLI (1970) e COUTINHO et al. (1982). A presença de titânio confere a coloração acastanhada aos piroxênios, que foram chamados de titâniopiroxênios por COUTINHO et al. (1982). Estes autores consideram que o nome desta rocha é analcimatinguaito.

SIAL \& McREATH (1984) descrevem a nefelina como produto de cristalização primária e também como produto da interação entre magmas basálticos e rochas carbonatadas, podendo, talvez, ser o caso das rochas alcalinas de Jaboticabal, pois o município se situa na regiåo de contato entre os sedimentos do Grupo Bauru e dos basaltos da Formação Serra Geral.

$\mathrm{O}$ tinguaito (Terciário) é mais recente que os basaltos da Formação Serra Geral de idade Cretácea. Isto pode ter influenciado sua ocorrência na paisagem (Figura 1), bem como o intemperismo diferencial, constatado na gênese dos latossolos. $O$ afloramento do tinguaito pode estar relacionado à áreas rejuvenescidas pela erosão, sendo que a renovação do material de origem dos solos permite $o$ aparecimento de perfis diversos, menos alterados, mais ricos em nutrientes.
Os solos estudados são bastante semelhantes do ponto de vista morfológico. $\mathrm{O}$ horizonte $\mathrm{Bw}$ possui textura argilosa; estrutura em bloco subangular, média, fraca; consistência ligeiramente dura, muito friável, plástica e pegajosa.

As TABELAS 1 e 2 apresentam, respectivamente, as características dos Latossolos Roxo formados sob a influência do basalto (LRd) e do tinguaito (LRe). Verifica-se que ocorreram variações nas características químicas, fisicas e mineralógicas dos mesmos. Os valores sugerem que o solo oriundo do basalto (LRd), sofreu alteraç̃̃es mais agressivas, sofrendo intensa decomposição dos constituintes iniciais e dos subsequentes integrantes do próprio solo. A relação molecular $\mathrm{Ki}$ mais baixa apresentada pelo LRd, indica que o mesmo é um latossolo sesquioxidico, ao passo que o LRe pode ser considerado como caulinítico, de acordo com a proposta feita por RESENDE \& SANTANA (1988), para estimar a mineralogia dos latossolos. A Tabela 3, ratifica esta consideração, pelos valores de caulinita encontrados na fração argila do $\mathrm{LRe}$ em relação ao LRd, sendo que a Figura 2 ilustra este resultado.

Os valores obtidos para extrações com ferro ditionito (Fed) e com oxalato (Feo), propiciaram relaçōes Feo/Fed, variando de 0,04 a 0,06 (TABELA 3), evidenciando a predominância de óxidos cristalinos nas argilas do LRe e LRd, a exemplo do que encontraram POMBO et al. (1982) para Latossolo Roxo oriundo de rocha basáltica.

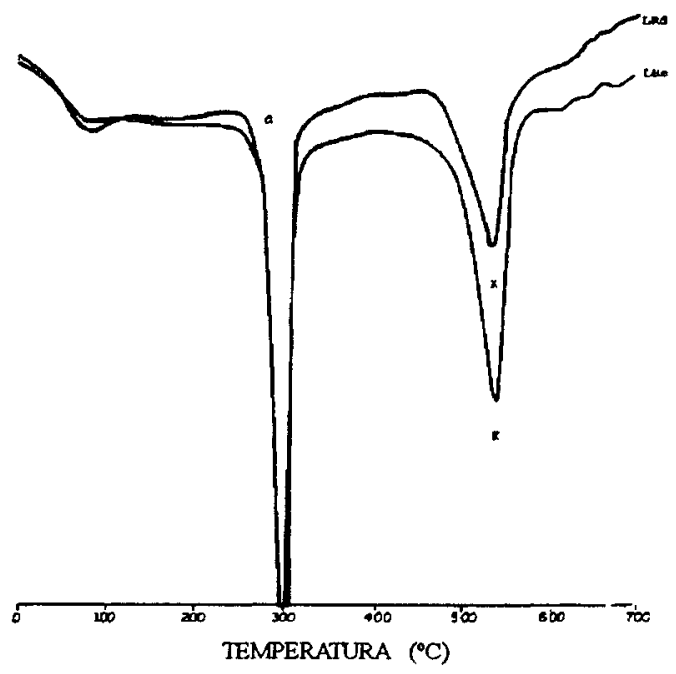

Figura 2 - Curvas de ATD da fração argila sem ferro. G - Gibbsita, K - Caulinita 
Os valores mais elevados encontrados no LRe referentes a relação silte/argila e saturação por bases, e os menores de argila e ferro total podem estar relacionados ao fato da rocha alcalina ser de ocorrência posterior ao basalto, portanto esteve menos tempo exposta à ação dos agentes de intemperismos. A presença de minerais mais resistentes ao intemperismo propiciou a formação de Latossolo Roxo eutrófico, unidade esta de ocorrência pouco frequente no Estado de São Paulo (OLIVEIRA \& MENK, 1984) e no Brasil (EMBRAPA, 1981). A Figura 3 reforça a hipótese levantada, mostrando que a CTC da fração argila do $\operatorname{LRe}(4,6 \mathrm{meq})$ é maior que aquela encontrada para o LRd $(0,4 \mathrm{meq})$.

Conclui-se que a ocorrência de Latossolo Roxo eutrófico no campus da UNESP-Jaboticabal, está associada a influência de rochas alcalinas, notadamente o tinguaito.

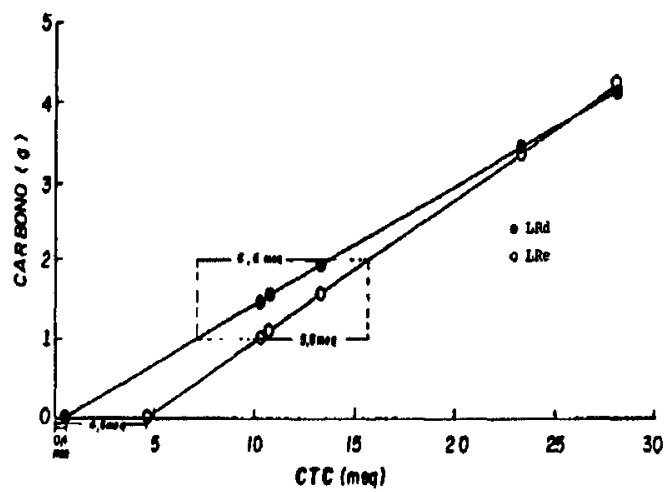

Figura 3 - Relação Carbono e CTC para $100 \mathrm{~g}$ de argila (Bennema, 1966), referentes ao perfil do LRd e LRe.

TABELA 1 Características fisicas e químicas do perfil do Latossolo Roxo originado sob a influência do basalto (LRd).

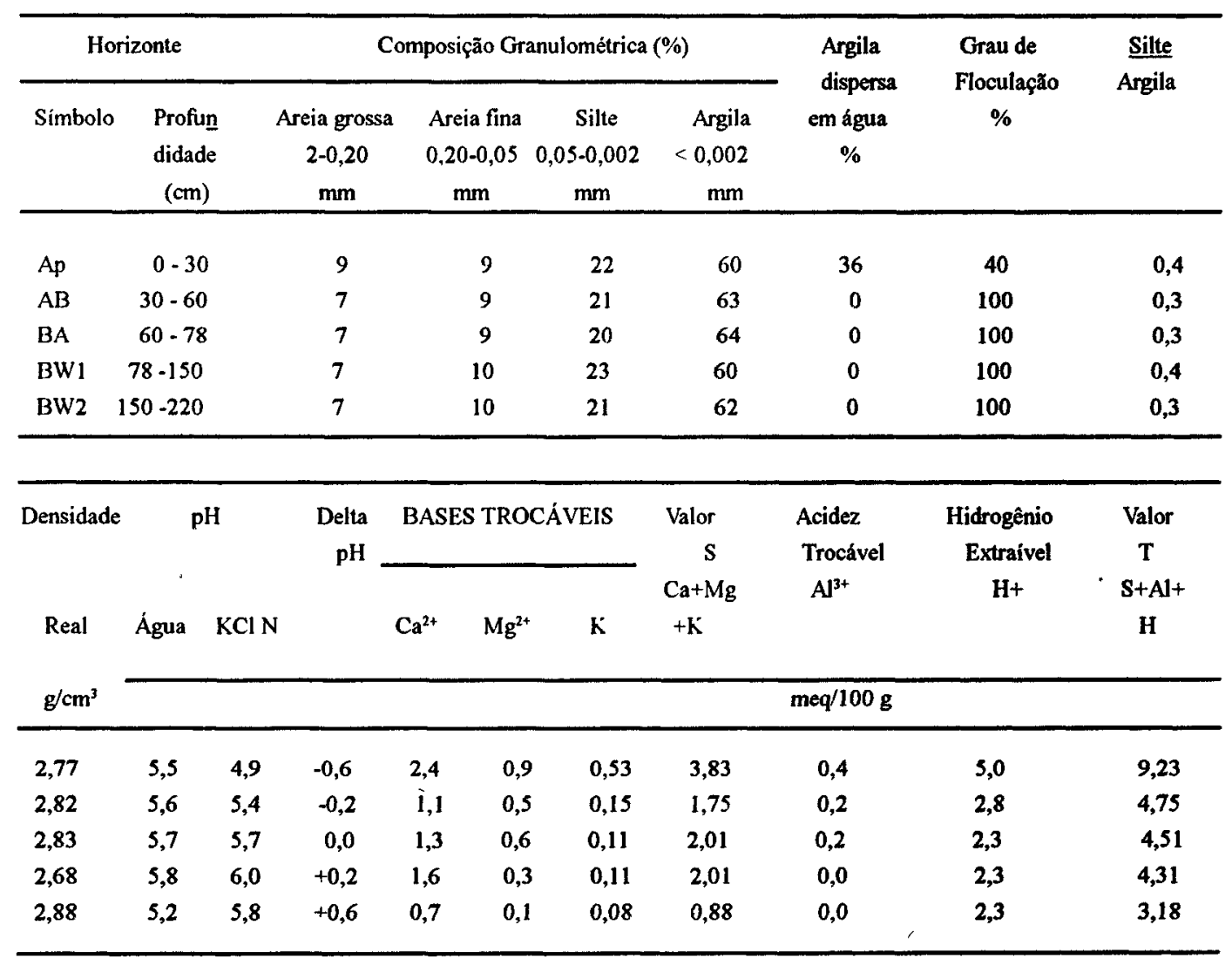


TABELA 1. Cont.

\begin{tabular}{|c|c|c|c|c|c|c|c|c|c|}
\hline \multirow{2}{*}{$\begin{array}{c}\text { Valor V } \\
100 . \mathrm{S} / \mathrm{T} \\
(\%)\end{array}$} & \multirow{2}{*}{$\begin{array}{l}\text { Sat.com aluminio } \\
100 .\left.A\right|^{3+/ S}+\left.A\right|^{3+} \\
(\%)\end{array}$} & \multirow{2}{*}{$\begin{array}{l}\text { MATÉRIA } \\
\text { ORGÂNICA } \\
\text { (\%) }\end{array}$} & \multicolumn{3}{|c|}{ ATAQUE SULFÚRICO } & \multirow{2}{*}{$\begin{array}{c}\mathrm{SiO}_{2} / \\
\mathrm{Al}_{2} \mathrm{O}_{3} \\
(\mathrm{Ki})\end{array}$} & \multirow{2}{*}{$\begin{array}{r}\mathrm{SiO}_{2} / \\
\mathrm{R}_{2} \mathrm{O}_{3} \\
(\mathrm{Kr})\end{array}$} & \multirow{2}{*}{$\begin{array}{r}\mathrm{Al}_{2} \mathrm{O}_{3} \\
\mathrm{Fe}_{2} \mathrm{O}_{3}\end{array}$} & \multirow{2}{*}{$\begin{array}{c}\text { P } \\
\text { Solúvel } \\
\text { ppm }\end{array}$} \\
\hline & & & $\begin{array}{l}\mathrm{SiO}_{2} \\
(\%)\end{array}$ & $\begin{array}{l}\mathrm{Al}_{2} \mathrm{O}_{3} \\
(\%)\end{array}$ & $\begin{array}{c}\mathrm{Fe}_{2} \mathrm{O}_{3} \\
(\%)\end{array}$ & & & & \\
\hline 42 & 9 & 2,4 & 11,5 & 23,5 & 20,5 & 0,83 & 0,53 & 1,80 & 66 \\
\hline 37 & 10 & 1,2 & 12,0 & 24,0 & 20,7 & 0,85 & 0,55 & 1,82 & 4 \\
\hline 45 & 9 & 1,1 & 12,0 & 26,5 & 22,2 & 0,77 & 0,50 & 1,87 & 3 \\
\hline 47 & 0 & 0,9 & 12,0 & 25,0 & 22,6 & 0,82 & 0,52 & 1,74 & 2 \\
\hline 28 & 0 & 0,8 & 9,8 & 26,0 & 21,9 & 0,64 & 0,42 & 1,86 & 2 \\
\hline
\end{tabular}

TABELA 2. Características fisicas e químicas do perfil do Latossolo Roxo originado sob a influência do tinguaito (LRe).

\begin{tabular}{|c|c|c|c|c|c|c|c|c|c|c|}
\hline \multicolumn{3}{|c|}{ Horizonte } & \multicolumn{5}{|c|}{ Composị̧ăo Granulométrica (\%) } & \multirow{2}{*}{$\begin{array}{c}\text { Argila } \\
\text { dispersa } \\
\text { em água } \\
\%\end{array}$} & \multirow{2}{*}{$\begin{array}{c}\text { Grau de } \\
\text { Floculaçăo } \\
\%\end{array}$} & \multirow{2}{*}{$\frac{\text { Silte }}{\text { Argila }}$} \\
\hline Símbolo & $\begin{array}{r}\text { Profun } \\
\text { didade } \\
(\mathrm{cm}) \\
\end{array}$ & & $\begin{array}{c}\text { Areia grossa } \\
2-0,20 \\
\mathrm{~mm}\end{array}$ & $\begin{array}{r}\text { Are } \\
0,20 \\
n\end{array}$ & $\begin{array}{l}\text { fina } \\
0,05 \\
m\end{array}$ & $\begin{array}{c}\text { Silte } \\
0,05-0,002 \\
\mathrm{~mm}\end{array}$ & $\begin{array}{r}\text { Argila } \\
<0,002 \\
\mathrm{~mm} \\
\end{array}$ & & & \\
\hline $\begin{array}{l}\text { Ap } \\
\text { AB } \\
\text { BA } \\
\text { BW1 } \\
\text { BW2 }\end{array}$ & $\begin{array}{r}0-26 \\
26-56 \\
56-97 \\
97-160 \\
160\end{array}$ & & $\begin{array}{l}7 \\
7 \\
7 \\
6 \\
6\end{array}$ & $\begin{array}{l}1 \\
1 \\
1 \\
1 \\
1\end{array}$ & & $\begin{array}{l}32 \\
29 \\
24 \\
27 \\
32\end{array}$ & $\begin{array}{l}46 \\
53 \\
59 \\
55 \\
50\end{array}$ & $\begin{array}{r}33 \\
37 \\
21 \\
0 \\
0\end{array}$ & $\begin{array}{r}28 \\
30 \\
64 \\
100 \\
100\end{array}$ & $\begin{array}{l}0,7 \\
0,5 \\
0,4 \\
0,5 \\
0,6\end{array}$ \\
\hline Densidade & Água & $\mathrm{KClN}$ & $\begin{array}{l}\text { Delta } \\
\text { pH }\end{array}$ & BASE & TROC & CÁVEIS & $\begin{array}{l}\text { Valor } \\
\quad \mathrm{S} \\
\mathrm{Ca}+\mathrm{Mg} \\
+\mathrm{K}\end{array}$ & $\begin{array}{c}\text { Acidez } \\
\text { Trocável } \\
\mathbf{A l}^{3+}\end{array}$ & $\begin{array}{c}\text { Hidrogênio } \\
\text { Extraivel } \\
\text { H+ }\end{array}$ & $\begin{array}{c}\text { Valor } \\
\mathbf{T} \\
\mathbf{S}+\mathbf{A} \mathbf{I}+ \\
\mathbf{H}\end{array}$ \\
\hline $\mathrm{g} / \mathrm{cm}^{3}$ & & & & & & & & $\mathrm{meq} / 10 \mathrm{~g}_{\mathrm{g}}$ & & \\
\hline $\begin{array}{l}2,73 \\
2,73 \\
2,74 \\
2,60 \\
2,78\end{array}$ & $\begin{array}{l}7,2 \\
7,1 \\
6,8 \\
6,6 \\
6,7\end{array}$ & $\begin{array}{l}6,5 \\
6,4 \\
6,2 \\
6,2 \\
6,4\end{array}$ & $\begin{array}{l}-0,8 \\
-0,7 \\
-0,6 \\
-0,4 \\
-0,3\end{array}$ & $\begin{array}{l}7,4 \\
8,1 \\
4,5 \\
3,2 \\
2,9\end{array}$ & $\begin{array}{l}3,2 \\
2,2 \\
0,9 \\
0,4 \\
0,6\end{array}$ & $\begin{array}{l}0,39 \\
0,22 \\
0,43 \\
0,06 \\
0,06\end{array}$ & $\begin{array}{r}10,94 \\
10,52 \\
5,83 \\
3,66 \\
3,56\end{array}$ & $\begin{array}{l}0 \\
0 \\
0 \\
0 \\
0\end{array}$ & $\begin{array}{l}2,0 \\
1,8 \\
2,0 \\
2,0 \\
1,8\end{array}$ & $\begin{array}{c}19,89 \\
12,32 \\
7,83 \\
5,66 \\
5,36\end{array}$ \\
\hline
\end{tabular}


TABELA 2. Cont.

\begin{tabular}{|c|c|c|c|c|c|c|c|c|c|}
\hline \multirow{2}{*}{$\begin{array}{c}\text { Valor V } \\
100 . \mathrm{S} / \mathrm{T} \\
(\%)\end{array}$} & \multirow{2}{*}{$\begin{array}{l}\text { Sat.com aluminio } \\
100 . \mathrm{Al}^{3+} / \mathrm{S}+\mathrm{Al}^{3+} \\
(\%)\end{array}$} & \multirow{2}{*}{$\begin{array}{r}\text { MATÉRIA } \\
\text { ORGÂNICA } \\
(\%)\end{array}$} & \multicolumn{3}{|c|}{ ATAQUE SULFÚRICO } & \multirow{2}{*}{$\begin{array}{r}\mathrm{SiO}_{2} / \\
\mathrm{Al}_{2} \mathrm{O}_{3} \\
(\mathrm{Ki})\end{array}$} & \multirow{2}{*}{$\begin{array}{r}\mathrm{SiO}_{2} \prime \\
\mathrm{R}_{2} \mathrm{O}_{3} \\
(\mathrm{Kr})\end{array}$} & \multirow{2}{*}{$\begin{array}{l}\mathrm{Al}_{2} \mathrm{O}_{3} / \\
\mathrm{Fe}_{2} \mathrm{O}_{3}\end{array}$} & \multirow{2}{*}{$\begin{array}{c}\text { P } \\
\text { Solúvel } \\
\text { ppm }\end{array}$} \\
\hline & & & $\begin{array}{l}\mathrm{SiO}_{2} \\
(\%)\end{array}$ & $\begin{array}{c}\mathrm{Al}_{2} \mathrm{O}_{3} \\
(\%)\end{array}$ & $\begin{array}{c}\mathrm{Fe}_{2} \mathrm{O}_{3} \\
(\%)\end{array}$ & & & & \\
\hline 85 & 0 & 3,5 & 11,2 & 24,3 & 17,2 & 0,78 & 0,54 & 2,22 & 17 \\
\hline 85 & 0 & 2,6 & 12,0 & 26,5 & 17,6 & 0,77 & 0,54 & 2,36 & 14 \\
\hline 74 & 0 & 1,4 & 13,8 & 28,0 & 17,9 & 0,84 & 0,60 & 2,45 & 12 \\
\hline 65 & 0 & 1,1 & 16,9 & 29,9 & 18,4 & 0,96 & 0,69 & 2,55 & 6 \\
\hline 66 & 0 & 1,1 & 17,2 & 27,5 & 19,0 & 1,06 & 0,74 & 2,27 & 3 \\
\hline
\end{tabular}

TABELA 3. Concentraç̃̃es de ferro obtidas através de ataque sulfúrico $\left(\mathrm{Fe}_{1}\right)$, extraídos com oxalato de amônio ( $\mathrm{Fe}_{\mathrm{o}}$ ) e ditionito-citrato-bicarbonato $\left(\mathrm{Fe}_{\mathrm{d}}\right)$ e relação $\mathrm{Fe}_{0} / \mathrm{Fe}_{\mathrm{d}}$ e $\mathrm{Fe}_{\mathrm{d}} / \mathrm{Fe}_{\text {, }}$, em amostras da fração argila. Valores de caulinita (C) e gibbsita $(G)$ obtidos na fração argila desferrificada.

\begin{tabular}{|c|c|c|c|c|c|c|c|c|}
\hline SOLO & HORIZONTE & $\mathrm{Fe}_{\mathrm{t}}$ & $\begin{array}{r}\mathrm{Fe}_{0} \\
-\% \\
\end{array}$ & $\mathrm{Fe}_{\mathrm{d}}$ & $\mathrm{Fe}_{\mathrm{o}} / \mathrm{Fe} \mathrm{e}_{\mathrm{d}}$ & $\mathrm{Fe}_{\mathrm{d}} \mathrm{Fe}_{\mathrm{t}}$ & C & $\begin{array}{c}\mathrm{G} \\
- \\
-\end{array}$ \\
\hline LRd & Bwl & 12,9 & 0,60 & 9,59 & 0,06 & 0,74 & 47,4 & 32,3 \\
\hline LRd & $\mathrm{Bw} 2$ & 14,6 & 0,64 & 10,45 & 0,06 & 0,72 & 35,9 & 34,5 \\
\hline $\mathrm{LRe}$ & Bwl & 17,9 & 0,66 & 12,43 & 0,05 & 0,69 & 63,0 & 34,8 \\
\hline $\mathrm{LRe}$ & $\mathrm{Bw} 2$ & 17,9 & 0,64 & 14,67 & 0,04 & 0,82 & 58,1 & 35,6 \\
\hline
\end{tabular}

\section{REFERENCIAS BIBLIOGRÁFICAS}

BARCHA, S.F.; ELLERT, N.; VALARELLI, J.V.; BONI, N.R. Intrusão alcalina de Jaboticabal, SP. Geociências, São Paulo, v.3, p.21-38, 1984.

CAMARGO, O.A. MONIZ, A.C.; JORGE, J.A.; VALADARES, J.M.A.S. Métodos de análise química, mineralógica e fisica de solos do Instituto Agronômico do Estado de São Paulo. Campinas: Instituto Agronômico, 1986. 94p. (Boletim Técnico, 106).

CENTRO NACIONAL DE ENSINO E PESQUISAS AGRONÔMICAS COM SOLOS. Levantamento de reconhecimento dos solos do

Estado de São Paulo: contribuição à carta de solos do Brasil. Rio de Janeiro: Comissão de Solos, 1960. (Boletim do Serviço Nacional de Pesquisas Agronômicas, 12).
COUTINHO, J.M.V.; COIMBRA, AM.; BRANDT NETO. M.; ROCHA, G.A.

Lavas alcalinas analcimíticas associadas ao grupo Bauru (Kb) no Estado de São Paulo, Brasil. In: CONGRESSO LATINO-AMERICANO DE GEOLOGIA, 5., Buenos Aires, 1982. p.133-142.

EMPRESA BRASILEIRA DE PESQUTSA -Serviço Nacional de Levantamento e Conservação de Solos. Manual de métodos de análise do solo. Rio de Janeiro, 1979. n.p.

EMPRESA BRASILEIRA DE PESQUISA -Serviço Nacional de Levantamento e Conservação de Solo. Mapa de solos do Brasil. Rio de Janeiro, 1981. n.p.

GUERRA, A.T. Dicionário geolbgico-geomorfológico. 3.ed. Rio de Janeiro: Fundação IBGE, 1969. 439p. (Publicação, 21). 
GOMES, C.B.; VALARELLI, J.V. Nova ocorrência de rochas alcalinas no Estado de Sho Paulo. In: CONGRESSO BRASILEIRO DE GEOLOGIA, 24., Brasilia, 1970. Resumos... Brasilia, Sociedade Brasileira de Geologia, 1970. p.336-337.

INSTTTUTO DE PESQUISAS TECNOLÓGICAS. Divisăo de Minas e Geologia Aplicada. Mapa de Javidas e ocorrências minerais do Estado de São Paulo. São Paulo, 1981. v.3.

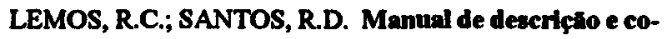
leta do solo no campo. Campinas: Sociedade Brasileira de Ciência do Solo, 1982. 46p.

MEHRA, O.P; JACKSON, M.L. Iron oxide removal from soil and clays by dithionite-citrate system buffered with sodium bicarbonate. Clays and Clay Minerals, New York, v.7, p.317-327, 1960.

OLIVEIRA, J.B.; MENK, J.R.F. Latossolos Roxos do Estado de Szo Paulo. Campinas: Instituto Agronômico, 1984. 135p. (Boletim Técnico, 82).

PINOTTI, R.F.; CORSINI, P.C.; MADUREIRA FILHO, J.B.; VALARELLI, J.V. Levantamento de solos contribuindo para descoberta geológica. In: CONGRESSO BRASILEIRO DE GEOLOGIA, 24., Brasília, 1970. Resumos... Brasília, Sociedade Brasileira de Geologia, 1970, p.59-60.

Sci. agric., Piracicaba, 52(2):226-232, mai./ago. 1995
POMBO, L.C.A; KLAMT, E.; KUNRATH, I.; GIANLUPPI, D. Identificaçăo de 6xidos de ferro na fraçăo argila de Latossolo Roxo. Revista Brasilleim de Ciência do Solo, Campinas, v.6, p.13-17, 1982.

RAIJ, B. van; QUAGGIO, J.A. Métodos de análise de solo para fins de fertilidade. Campinas, Instituto Agronômico, 1983. 31p. (Boletim Técnico, 81).

RESENDE, M; SANTANA, D.P. Uso das relaçres Ki e Kr na estimativa da mineralogia para classificação dos latossolos. In: REUNIÃO DE CLASSIFICAÇÃO, CORRELAÇÃO DE SOLOS E INTERPRETAÇÃO DE APTIDẢO AGRÍCOLA, 3., Anais... Rio de Janeiro, EMBRAPA/SNLCS, 1988. p.225-232, (Documento SNLCS, 12).

ROTTA, C.L. Noçðes Gerais de Geologia. In: MONIZ, A.C., coord. Elementos de Pedologia. São Paulo, EDUSP, 1972. p.289-303.

SÃo PAULO. Departamento de águas e energia elétrica. Estudo de águas subterrîness: Regỉo Administrativa 6, Ribeiräo Preto, São Paulo, DAEE, 1974. v.1.

SIAL, A.N.; McREATH, I. Petrologia Ignea: os fundamentos e as ferramentas de estudos. Salvador, Bureau Gráfica, 1984. 167p.

Recebido para publicação em 12.07.94

Aceito para publicação em 20.11.94 